\title{
Temperature Rising at the Discontinuous Surface of Plasma
}

(Received November 2, 1967)

A consideration is given to the phenomenon of temperature rising at a discontinuous level where a normal fluid in a steady state and a superfluid of plasma stream collide and mix.

In this field, it is possible to work out a calculation as to the propagation circumstances of temperature differentiated in the two types of particles motion by coupling them in one equation, when the latter type propagating in a sine curve seems to approximate the former that constitutes a broad straight line.

The temperature waves exist between the superfluid stream and the normal fluid of the steady state. This wave has been introduced by L. Tisza as the "Two Fluid Model" and was named the second sound wave. The equation of temperature wave for the boundary surface of two fluids is written in the form

$$
\frac{\partial^{2} T}{\partial t^{2}}-\frac{S^{* 2} T}{C_{p}} \frac{\partial^{2} T}{\partial z^{2}}=0
$$

where $\stackrel{*}{S}$ is the constant entropy transported by the shock stream. The velocity of the temperature wave is $\stackrel{*}{S} \sqrt{\frac{T}{C_{p}}}$, where $C_{p}$ is the specific heat at constant pressure. In equation (1) we have taken pressure, density as constants respectively and the temperature as variable. This depends upon the fact that the particles always flow into the normal part of the fluid stream from the superfluid part of the different medium. On the other hand, when the heat is increasing we have the following general equation

$$
\frac{\partial T}{\partial t}=\frac{k}{\rho C} \frac{\partial^{2} T}{\partial z^{2}}
$$

where $k$ is the degree of the heat conduction and $\frac{k}{\rho C} \equiv K$ is a thermal diffusibility. From eq. (1) and (2) we obtain

$$
\frac{\partial^{2} T}{\partial t^{2}}-M \cdot T \frac{\partial T}{\partial t}=0
$$

where $M \equiv \frac{S^{* 2}}{K C_{p}}$. This equation indicates the mutual action of the thermal particles between the temperature wave caused by the superfluid and the heat translation in the boundary surface of the two mediums.

And its equation is considered as the time variation of the temperature in the normal stationary stream into which the superfluid stream flowed. From eq. (3) we have $\frac{\partial T}{\partial t}=$ $\frac{1}{2} M T^{2}+C_{1}^{*}(z)$, where $C_{1}^{*}(z)$ is the value of $\frac{\partial T}{\partial t}$ when $t=0$. When the position is fixed we 
have

$$
\frac{2}{M} \int \frac{d T}{T^{2}+C_{2}^{*}(z)}=\int d t
$$

where

$$
C_{2}^{*}(z)=\frac{2}{M} C_{1}^{*}(z) \text {. }
$$

From equation (4), when $C_{2}^{*}(z)>0$, we have $\frac{2}{M} \int \frac{d T}{T^{2}+A^{2}}=\int d t$

where

$$
A^{2}=C_{2}^{*}(z) \text {. }
$$

From the relation (5), we have $\frac{2}{M} \frac{1}{A} \tan ^{-1} \frac{T}{A}=t+t_{0}^{*}$,

where $t_{0}^{*}$ is an integral constant.

Hence $\quad t=\frac{2}{M A}\left(\tan ^{-1} \frac{T}{A}-\tan ^{-1} \frac{T_{0}}{A}\right)$, where $T_{0}$ is the value of $T$ when $t=0$.

Therefore we have

$$
T=A \frac{\sin \left(\frac{M A}{2} t+\varepsilon_{1}\right)}{\sin \left(\frac{M A}{2} t+\varepsilon_{2}\right)},
$$

where

$$
\tan \varepsilon_{1}=\frac{T_{0}}{A}, \tan \varepsilon_{2}=\frac{A}{T_{0}} \text {. }
$$

Also

$$
A=\sqrt{C_{2}^{*}(z)}=\sqrt{\frac{2}{M} C_{1}^{*}(z)}=\sqrt{\frac{2}{M}\left(\frac{\partial T}{\partial t}\right)_{0}} .
$$

$\left(\frac{\partial T}{\partial t}\right)_{0}$ is the value of $\frac{\partial T}{\partial t}$ when $t=0$.

According to the result of the relation of the temperature variations with $t$ at the discontinuous place of fluids, if the initial tendency of the variation of the temperature is very slow, the generated temperature at this place will rapidly increase.

\section{Yoshihito TAKESADA}

Kyoto University of Education

Fushimi-ku, Kyoto, Japan 